\title{
Frequencies of RhCE and Kell phenotypes in Xinjiang using a cross-minorities transfusion simulation model
}

\author{
Wei Chen ${ }^{1}$, Jun Wen ${ }^{1}$, Fei Li ${ }^{1}$, Changmin Wang ${ }^{2 *}$, Qing Li ${ }^{3}$, Gang Zhao ${ }^{3}$ \\ ${ }^{1}$ Department of Blood Transfusion, Xinjiang Uyghur Autonomous People's Hospital, Xinjiang Medical University,Urumqi, Xinjiang, \\ 830001, China; \\ ${ }^{2}$ Clinincal Laboratory, Xinjiang Uyghur Autonomous People's Hospital, Urumqi, Xinjiang, 830001, China; \\ ${ }^{3}$ Department of Blood Transfusion, Affiliated Hospital of Traditional Chinese Medicine of Xinjiang Medical Uninersity, Urumqi,
} Xinjiang, 834000, China

\begin{abstract}
Xinjiang represents one of the richest minorities' areas in China. This high ethnic diversity reflects in the blood groups and immune status and has a consequent impact on blood transfusions. To evaluate the risks of crossminority transfusion in Xinjiang, we investigated the frequencies of erythrocytic Rh and $\mathrm{K}$ antigens among 1,073 Uyghurs and 213 Kazaks. We further reviewed the literature on the frequency of erythrocytic antigens to develop a simulation model for calculating the risk of patients in Xinjiang exposed to mismatched erythrocytic antigens. The frequencies of $\mathrm{RhE}, \mathrm{RhC}$, and $\mathrm{K}$ phenotypes were as follows: $\mathrm{C}$ antigen, $52.3 \%$ in Uyghurs and $56.8 \%$ in Kazaks; c antigen, $47.7 \%$ in Uyghurs and $43.2 \%$ in Kazaks; E antigen, 25.5\% in Uyghurs and 27.2\% in Kazaks; e antigen, $74.5 \%$ in Uyghurs and $72.8 \%$ in Kazaks; $\mathrm{K}$ antigen, $1.8 \%$ in Uyghurs and $1.8 \%$ in Kazaks. The population-adjusted cumulative match rate demonstrated that $53.3 \%, 51.4 \%, 50.6 \%$, and $53.7 \%$ of the Uyghur, Kazak, Han, and Hui populations were recipients, respectively, although the recipients were transfused with an unknown $\mathrm{Rh}$ blood type. We concluded that the risks of cross-minority transfusion in Xinjiang are insignificant. The best strategy appears to be $\mathrm{K}$ and $\mathrm{Rh}-$ matched transfusions in this region due to the much higher frequency of the $\mathrm{K}$ antigen compared toother areas in China.
\end{abstract}

Keywords: Kell(K) blood type, $\mathrm{Rh}(\mathrm{CE})$ blood type, Uyghur, Kazak, antigen distribution

\section{INTRODUCTION}

Xinjiang is the one of the richest minority areas in China with over 13 ethnicities living in various areas, including Han, Hui, Uyghur, Manchu, Kirgiz, Tajik, Xib, Ozbek, Russian, Tatar, Mongolian, Kazak, and Daur. According to the 6th China Census conducted in 2010, four ethnicities represented over $95 \%$ of the population in Xinjiang, these were 11.27 million $\mathrm{Uy}-$ ghurs(48.53\%), 8.60 million Hans(37.01\%), 1.60 mil- lion $\operatorname{Kazaks}(6.88 \%)$, and 1.06 million Huis $(4.56 \%)^{[1]}$.

Recent studies confirmed that red blood cell(RBC) allo-antibodies against the Rh system were the major allo-antibodies in China ${ }^{[2]}$. Several studies have demonstrated a high diversity in the frequency of RBC allo-antibodies among Hans and Uyghurs ${ }^{[3]}$. Moreover, the numbers of antibodies against Asian-specific high-frequency antigens, including $\mathrm{Fy}^{\mathrm{a}}$, and lowfrequency antigens such as $\mathrm{K}$ are reported to be notably high in Xinjiang ${ }^{[3-4]}$. Although the frequencies of blood groups, including $\mathrm{ABO}, \mathrm{Rh}$, Kidd, and MNS in

*Correspondence to: Changmin Wang, Clinincal Laboratory, Xinjiang Uyghur Autonomous People' s Hospital, Urumqi, Xinjiang, 830001, China. TEL: 86-991-8564491; E-mail: wcm224@126.com.

${ }^{\Delta}$ Theseauthors contributed equally to this work. 
Xinjiang have been reported, some blood groups have still as yet not beeninvestigated in this region, including the Kell system and anti-K, which are known to show a higher prevalence in Xinjiang compared to that in Beijing ${ }^{[4]}$. Despite the ethnic diversity of blood groups however, and their potential immune responses impact on transfusion, few studies have evaluated the status of cross-minorities' transfusions in Xinjiang.

To evaluate the risks of cross-minority transfusions in Xinjiang, we investigated the frequencies of erythrocytic $\mathrm{Rh}$ and $\mathrm{K}$ antigens among the major minority groups: Uyghurs and Kazaks.We further reviewed the literature to obtain the reported frequencies of erythrocytic antigens, which were used to conduct a simulation study for determining the risk of exposure to mismatched erythrocytic antigens for patients in Xinjiang.

\section{MATERIALS AND METHODS}

\section{Study population}

The subjects enrolled in our study were recruited from two medical centres, Xinjiang Uyghur Autonomous People' s Hospital and the Affiliated Hospital of Traditional Chinese Medicine of Xinjiang Medical University, between June 2016 and December 2016. Subjects with a history of transfusion in the last three months or auto-controlled reported positive specimens were excluded. For subjects that had been admitted at either or both of the medical centres several times during the period of the study, only the data from the first report were recorded. Overall, 1,073 Uyghurs, including 529 males (49.3\%) and 544 females (50.7\%), and 213 Kazaks, including 96 males (45.1\%) and 117 females (54.9\%),were included in the study and phenotyped for $\mathrm{RhCE}$ and Kell. The age ranges were 5 days to 85 years in Uyghurs and 12 days to 78 years in Kazaks.

To calculate the risks of exposure to mismatched erythrocytic antigens for the patients in Xinjiang, we reviewed available studies ${ }^{[5,6]}$. The reported frequencies of RhCE phenotypes were as follows: $45.1 \%$ CCee, $1.9 \%$ CCEe, $0.0 \%$ CCEE, $9.1 \%$ Ccee, $26.6 \%$ CcEe, $3.8 \%$ CcEE, $1.9 \%$ ccee, $2.4 \%$ ccEe, and $9.1 \%$ ccEE in Hans; $33.0 \%$ CCee, $1.3 \%$ CCEe, $0.2 \%$ CCEE, $14.7 \%$ Ccee, $31.2 \%$ CcEe, $3.4 \%$ CcEE, $1.8 \%$ ccee, $8.3 \%$ ccEe, and $6.2 \%$ ccEE in Kazaks; $31.1 \%$ CCee, $2.2 \%$ CCEe, $0.2 \%$ CCEE, $19.1 \%$ Ccee, $25.4 \%$ CcEe, $1.8 \% \mathrm{CcEE}, 3.0 \%$ ccee, $8.1 \% \mathrm{ccEe}$, and $8.9 \% \mathrm{ccEE}$ in Uyghurs; and $37.1 \%$ CCee, $6.0 \%$ CCEe, 0.4\% CCEE, $9.2 \%$ Ccee, $30.7 \%$ CcEe, $1.2 \%$ CcEE, $2.0 \%$ ccee, $5.2 \% \mathrm{ccEe}$, and $8.4 \% \mathrm{ccEE}$ in the Hui minority.

\section{Phenotyping}

Three millilitres of peripheral venous blood was drawn from each subject and placed in EDTA- $\mathrm{Na}_{2}$ tubes. The phenotyping methods included a microbeads test for RhCE and $\mathrm{K}$ antigen performed on an automated machine (ORTHO Workstation, AutoVue, Ortho Clinical Diagnostics, UK). In addition, the samples were incubated with monoclonal antibodies against the erythrocytic antigens anti-C, anti-c, antiE, anti-e (Shanghai blood Biological Medicine Co Ltd., Shanghai, China), and anti-K (Sunquin GmbH, Switzerland). In brief, EDTA-whole blood was centrifuged at $1,000 \mathrm{~g}$ for $5 \mathrm{~min}$ (KUBOTA KA-2200, Japan) and $1 \mathrm{~mL}$ of packed RBCs was pipetted and centrifuged at $800 \mathrm{~g}$ for $1 \mathrm{~min}$ (KUBOTA MC-450, Japan); the procedure was repeated three times. Ten microliters of the washed packed RBCs was dissolved into $1 \mathrm{~mL}$ of $0.9 \%$ normal saline at a concentration of $0.8 \%-1.0 \%$. The RBC suspension was examined with $\mathrm{Rh} / \mathrm{K}$ micro-bead phenotyping kits $(\mathrm{Rh} / \mathrm{K}$ blood group diagnostic reagent card, Ortho BioVue System, Ortho Clinical Diagnostics, UK). For the tube method of analysis, $3 \%$ of the washed RBCs were reacted with $100 \mu \mathrm{L}$ of the monoclonal antibody by incubating at room temperature for $10 \mathrm{~min}$, and a positive $\mathrm{K}$ phenotype was judged when the titre of any phenotype was lower than $3+$. The other procedures such as antibody screening, sample reception, report documentation, and report writing were all based on regulations and the standard procedures specified by the transfusion departments of the two medical centres.

\section{Statistical analysis}

For the frequencies of RHCE alleles based on the RhCE phenotyping results, the chi-squared test was used to determine the fit to Hardy-Weinberg equilibrium. Differences between groups with a two-sided $P<0.05$ were considered statistically significant. All statistical analyses were conducted using SPSS version 16.0 (SPSS Inc., Chicago, IL, USA).

\section{RESULTS}

\section{Frequencies of RhCE phenotypes and alleles in Kazaks and Uyghurs}

The frequencies of RHCE alleles in Uyghurs were as follows: $26.6 \%$ CCee, $0.4 \% \mathrm{CCEe}, 0.1 \% \mathrm{CCEE}$, $21.8 \%$ Ccee, $28.4 \%$ CcEe, $0.4 \%$ CcEE, $6.9 \%$ ccee, $9.6 \% \mathrm{ccEe}$, and $5.9 \% \mathrm{ccEE}$, representing an insignificant difference $(P=0.999)$ from previously reported values. The frequencies of RHCE alleles in Kazaks were as follows: $34.7 \%$ CCee, $0.5 \%$ CCEe, $0.0 \%$ CCEE, $16.4 \%$ Ccee, $26.8 \%$ CcEe, $0.0 \%$ CcEE, $4.7 \%$ ccee, $6.6 \% \mathrm{ccEe}$, and $10.3 \% \mathrm{ccEE}$, also representing an insignificant difference $(P=1.000)$ with the pre- 
viously reported results (Table 1). The chi-squared test confirmed that the distribution of RHCE alleles in Uyghurs and Kazaks were in line with the HardyWeinberg equilibrium.

The frequencies of the RhC, RhE, and $\mathrm{K}$ phenotypes in Uyghurs were $52.3 \% \mathrm{C}$ antigen and $47.7 \%$

Table 1 Frequencies of $R h C E$ alleles in Uyghurs and Kazaks

\begin{tabular}{lrrrrrrrrr}
\hline & \multicolumn{3}{c}{ Uyghurs $(n=1,073)$} & \multicolumn{5}{c}{ Kazaks $(n=213)$} \\
Allele & \multicolumn{2}{c}{ Our study } & Previous & $P$ & \multicolumn{3}{c}{ Our study Previous } & Purl \\
& \multicolumn{1}{c}{$n$} & \multicolumn{1}{c}{$\%$} & study/\% & $P$ & \multicolumn{1}{c}{$n$} & $\%$ & study/\% & $P$ \\
\hline CCee & 285 & 26.6 & 31.1 & 0.999 & 74 & 34.7 & 33.0 & 1.000 \\
CCEe & 4 & 0.4 & 2.2 & & 1 & 0.5 & 1.3 & \\
CCEE & 1 & 0.1 & 0.2 & & 0 & 0.0 & 0.2 & \\
Ccee & 234 & 21.8 & 19.1 & & 35 & 16.4 & 14.7 & \\
CcEe & 305 & 28.4 & 25.4 & & 57 & 26.8 & 31.2 & \\
CcEE & 4 & 0.4 & 1.8 & & 0 & 0.0 & 3.4 & \\
ccee & 74 & 6.9 & 3.0 & & 10 & 4.7 & 1.8 & \\
ccEe & 103 & 9.6 & 8.1 & & 14 & 6.6 & 8.3 & \\
ccEE & 63 & 5.9 & 8.9 & & 22 & 10.3 & 6.2 & \\
\hline
\end{tabular}

Cantigen of RhC; $25.5 \%$ E antigen and $74.5 \%$ e antigen of RhE; and $1.8 \% \mathrm{~K}$ antigen of Kell. The frequencies of $\mathrm{RhC}, \mathrm{RhE}$, and $\mathrm{K}$ phenotypes in Kazaks were $56.8 \% \mathrm{C}$ antigen and $43.2 \% \mathrm{c}$ antigen of $\mathrm{RhC}$; $27.2 \% \mathrm{E}$ antigen and $72.8 \%$ e antigen of RhE; and $1.8 \% \mathrm{~K}$ antigen of Kell (Table 2).

\section{Rate of Rh-type compatibility between re- cipients and donors for minorities}

According to the frequencies of RHCE alleles in minorities living in Xinjiang, the rates of compatibility for patients with any Rh type among Xinjiang
Table 2 Frequencies of RhCE and Kell phenotypes in Uyghurs and Kazaks

\begin{tabular}{lcccc}
\hline \multirow{2}{*}{ Phenotype } & \multicolumn{2}{c}{ Uyghurs } & \multicolumn{2}{c}{ Kazaks } \\
& frquency & $\%$ & frquency & $\%$ \\
\hline RhC & & & & \\
$\mathrm{C}$ & 1,122 & 52.3 & 242 & 56.8 \\
$\mathrm{c}$ & 1,024 & 47.7 & 184 & 43.2 \\
$\mathrm{RhE}$ & & & & \\
$\mathrm{E}$ & 547 & 25.5 & 116 & 27.2 \\
$\mathrm{e}$ & 1,599 & 74.5 & 310 & 72.8 \\
$\mathrm{Kell}$ & & & & \\
$\mathrm{K}^{+}(\mathrm{Kk} / \mathrm{KK})$ & 19 & 1.8 & 4 & 1.8 \\
\hline
\end{tabular}

transfusion donors are shown in Table 3. Considering the minorities acting as recipients, the patients that received transfused blood from donors of Han ethnicity showed the highest cumulative match rate among the four major minorities in Xinjiang. The population-adjusted cumulative match rates were $53.3 \%, 51.4 \%, 50.6 \%$, and $53.7 \%$ for Uyghurs, $\mathrm{Ka}-$ zaks, Hans, and Huis as recipients. The recipients transfused with blood of an unknown Rh type and demographic composition based on the 6th China Census of 2010, were included as adjusted parameters (Table 4).

\section{DISCUSSION}

In this study, we determined the frequency of RHCE alleles and phenotypesof RhCE and the $\mathrm{K}$ antigen, and combined data from previous studies to analyze the transfusion-compatible rate with respect to RhCE matching for evaluating the risk of cross-

Table 3 Patients of different Rh types in Xinjiang transfused with $\mathrm{Rh}$-compatible blood from minorities (\%)

\begin{tabular}{|c|c|c|c|c|c|c|c|c|c|}
\hline \multirow{2}{*}{ Recipient } & \multicolumn{9}{|c|}{ Donor } \\
\hline & CCee & $\mathrm{CCEe}$ & $\mathrm{CCEE}$ & Ccee & $\mathrm{CcEe}$ & $\mathrm{CcEE}$ & ccee & $\mathrm{ccEe}$ & $\mathrm{ccEE}$ \\
\hline \multicolumn{10}{|l|}{ Alleles } \\
\hline CCee & Compatible & Compatible & & Compatible & Compatible & & & & \\
\hline CCEe & & Compatible & & & Compatible & & & & \\
\hline CCEE & & Compatible & Compatible & & Compatible & Compatible & & & \\
\hline $\mathrm{CcEe}$ & & & & & Compatible & & & & \\
\hline $\mathrm{CcEE}$ & & & & & Compatible & Compatible & & & \\
\hline ccee & & & & Compatible & Compatible & & Compatible & Compatible & \\
\hline $\mathrm{ccEe}$ & & & & & Compatible & & & Compatible & \\
\hline $\mathrm{ccEE}$ & & & & & Compatible & Compatible & & Compatible & Compatible \\
\hline Hans & 45.1 & 47.0 & 0.0 & 56.1 & 100.0 & 12.9 & 1.9 & 13.4 & 9.1 \\
\hline Kazaks & 34.7 & 35.2 & 0.0 & 55.8 & 100.0 & 10.3 & 4.7 & 21.6 & 10.3 \\
\hline Uyghurs & 26.6 & 27.1 & 0.1 & 55.3 & 100.0 & 6.4 & 6.9 & 22.4 & 5.9 \\
\hline Huis & 37.1 & 43.4 & 0.4 & 48.2 & 100.0 & 10.0 & 2.0 & 15.5 & 8.4 \\
\hline
\end{tabular}

The frequency of RhCE in monorities from our investigation and present study were regard as donor to offer the RhCE-match blood for recipients 
Table 4 Cumulative match rate between minorities as recipients and donors $(\%)$

\begin{tabular}{lcccc}
\hline \multirow{2}{*}{ Donor } & \multicolumn{4}{c}{ Recipient } \\
& Uyghurs & Kazaks & Hans & Huis \\
\hline Hans & 54.8 & 53.8 & 54.7 & 57.0 \\
Kazaks & 53.0 & 50.9 & 50.0 & 52.9 \\
Uyghurs & 50.7 & 47.7 & 45.6 & 49.1 \\
Huis & 51.1 & 49.7 & 50.1 & 53.1 \\
$\begin{array}{l}\text { Population-adjusted } \\
\text { cummulative match rate }\end{array}$ & 53.5 & 51.4 & 50.6 & 53.7 \\
\hline
\end{tabular}

Population-adjusted cumulative match rates were based on the minorities rate as an adjusted parameter according to the 6th China Census of 2010 .

minorities'transfusion ${ }^{[3]}$.

The frequencies of blood groups show diversity among races and areas. The prevalence of $\mathrm{K}$ antigen in the Kell blood groups was reported to be $0.02 \%-$ $0.48 \%$ in Japan, $5.68 \%$ in Indian blood donors, $9.8 \%$ in Caucasians, and $2 \%$ in Black donors ${ }^{[7-8]}$. In China, the prevalence of Chinese blood donors in Shanghai was reported to be $0.06 \%$, and the genotyping prevalence in the Huis and Uyghurs was $1.36 \%$ and $3.16 \%$, respectively, in Xinjiang ${ }^{[9-10]}$. Evers and Schonewille et $a l .{ }^{[11-12]}$ demonstrated donor-recipient RBC matching strategies will be most efficient when primarily focusing on the prevention of $\mathrm{C}, \mathrm{c}, \mathrm{E}, \mathrm{K}$, and $\mathrm{Jk}(\mathrm{a})$ alloimmunisation. Given that $\mathrm{K}$ shows adominant pattern of inheritance, the offspring from ethnic intermarriage between Hans and minorities can result in anti-Krelated haemolytic disease in newborns; therefore,the risks of cross-minorities' transfusion should be carefully considered ${ }^{[12]}$. A strategy of $\mathrm{Rh}$ and $\mathrm{K}$-matched transfusion may be possible because of the high $\mathrm{K}$ prevalence in Xinjiang, which is higher than that of populations in central China. In addition, the presence of anti-K has also been reported in Xinjiang.

According to the simulated model, it is easier to find compatible blood from Hans for patients who reported RBC allo-antibodies in Xinjiang with CCee, $\mathrm{CCEe}$, Ccee, and $\mathrm{CcEE}$ alleles. In addition, Kazak patients with ccEE, Uyghur patients with ccee and ccEe, and Hui patients with CCEE have a greater chance to find compatible blood than others of the same minorities. These conclusions are also supported by the ethic polymorphism of RBC blood groups, because of a previous government policy forcing Hans to migrate to Xinjiang around 100 years ago. Our simulation model indicates that cross-minority transfusion does not have a significant risk, and that Hans are the most appropriate blood donors regardless of the recipient minority type found in Xinjiang when the RhCE type of the recipients and donors are unknown.
Overall, we conclude that there is an insignificant risk of cross-minority transfusion in Xinjiang, and the best strategy is to perform $\mathrm{K}$ and $\mathrm{Rh}$-matched transfusion in Xinjiang due to the much higher frequency of the $\mathrm{K}$ antigen in this region compared to other districts in China.

\section{References}

[1] National Bureau of Statistics of China. The Sixth National Population Census Bulletin for Xinjiang District. http://www.stats.gov.cn/ztjc/zdtjgz/zgrkpc/dlcrkpc/dlcrkpcss/201105/ t20110506_69978.htm

[2] Lin YS, Chang JS, Qiu Y. Incidence of unexpected red blood cell antibodies in the north of China. Asia-Pacific Journal of Blood Types and Genes, 2017,1(1):17-24.

[3] Song XC, Liu B, Xu J, et al. Irregular antibody screening of Rh blood system and clinical safety transfusion for diverse minorities in Xinjiang. J Clin Hematol (In Chinese), 2016, 29(12): 962-4.

[4] Song XC, Liu B, Liu YJ, et al. Irregular antibody screening and clinical safety transfusion for diverse minorities in Xinjiang. J Clin Hematol (In Chinese), 2015, 28(10): 1272-4.

[5] Qiu F, GLBHT, Tian X. The Rh Distribution among minorities in Xinjiang. J Clin Transfus Lab Med(In Chinese), 2013, 15(3): 276-8.

[6] Mao Q, Zhao X, Liu J. The distribution of ABO and Rh in Naning. Chongqing Medicine, 2015, 44(10):1381-5.

[7] Marli ALRF, Jeane ELV,Gláucia ASG, et al.Rh, Kell, Duffy, Kidd and Diego blood group system polymorphism in Brazilian Japanese descendants. Transfus Apher Sci, 2014, 50(1): 123-8.

[8] Beenu T, Karan S, Ratti RS, et al.Phenotype frequencies of blood group systems (Rh, Kell, Kidd, Duffy,MNS, $\mathrm{P}$, Lewis, and Lutheran) in north Indian blood donors. Transfus Apher Sci, 2010, 43 (1): 17-22.

[9] Lin GY, Du XL, Shan JJ, et al. Molecular genetic analysis of genes from MNS, Duffy and Kell blood groups in the China Xinjiang Uygur population. Chinese Journal of Tissue Engineering Research(In Chinese), 2016, 20(1): 123-7.

[10] Lin G, Shan J, Zhang Y. Distribution of gene frequencies of 9 kinds of rare blood group system in Hui nationalities in Xinjiang, China. Journal of Xinjiang Medical University(In Chinese), 2016, 39(8): 1026-31.

[11] Evers D, Middelburg RA, de Haas M. Red-bloodcell alloimmunisation in relation to antigens' exposure and their immunogenicity: a cohort study.Lancet Haematol,2016,3(6):e284-92.

[12] Schonewille H, Honohan Á, van der Watering LM. Incidence of alloantibody formation after ABO-D or extended matched red blood cell transfusions: a randomized trial (MATCH study).Transfusion, 2016,56(2):311-20.

(Received 09 August 2017, Revised 11 September 2017, Accepted 16 September 2017) 